boost the immune attack against tumours, the authors suggest.

Science http://doi.org/bc7p (2016)

NEUROSCIENCE

\section{Altered sensations in anxiety}

Anxiety disorders could involve not only cognitive, but also sensory changes in the brain.

Recent studies have suggested that people with anxiety, after learning a negative stimulus, respond negatively to similar but neutral stimuli more often than healthy people. Rony $\mathrm{Paz}$ at the Weizmann Institute of Science in Rehovot, Israel, and his colleagues found that individuals with anxiety disorders also perceive these stimuli less precisely than healthy people do. After learning to associate a tone with either monetary gain or loss, participants were asked to decide whether a series of other sounds were a match to the previous ones or were new. People with anxiety disorders mistook a wider range of frequencies for the tones they had learned, compared with healthy people. Learned tones and neighbouring sounds triggered brain activity that showed greater similarity in people with the disorders than in healthy people. This effect was in the brain's auditory cortex and in the amygdala, which processes fear.

The findings suggest that people with anxiety have

al altered perception of certain stimuli, the authors say.

Curr. Biol. http://doi.org/bc3z (2016)

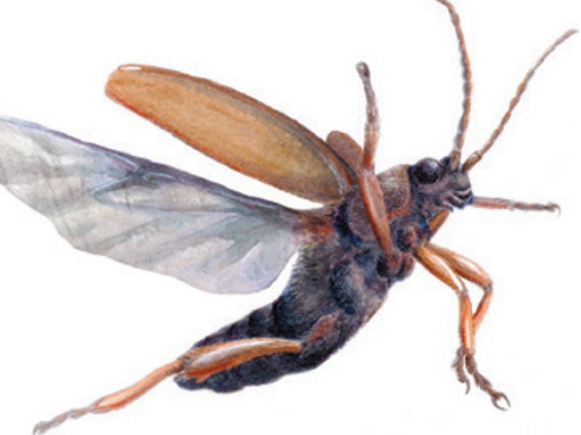

\section{Bacterial toxins invite infections}

Certain pneumonia-causing bacteria produce compounds that help other pathogenic bacteria to spread through the lungs.

Bret Sellman at

MedImmune, a biotechnology

firm in Gaithersburg,

Maryland, and his colleagues infected mice with a variety of bacterial species, either individually or in combination with Staphylococcus aureus, which can cause respiratory and other infections. Mice that were co-infected with S. aureus had higher levels of both microbe species in their lungs, and were more likely to die than animals infected with a single pathogen. The team found that a protein produced by $S$. aureus, called $\alpha$-toxin, aids the growth of several bacterial species by impairing immunecell function. Early treatment with an antibody against a-toxin helped to eliminate S. aureus and prevented other pathogens from multiplying.

The authors suggest that antibody-based treatments targeting a single bacterial species could help some people who are infected with multiple pathogens.

Sci. Transl. Med. 8, 329ra31 (2016)

\section{BIOMECHANICS \\ How flying beetles waterski}

The waterlily beetle exploits properties of the interface where air and water meet to glide quickly across the surface of ponds.

Manu Prakash at Stanford University in California and his colleagues filmed waterlily beetles (Galerucella nymphaeae; pictured) at 3,000 frames per second to characterize the mechanics of the insect's unusual mode of flight on the two-dimensional surface. They found that the claws on the beetles' legs remain submerged during

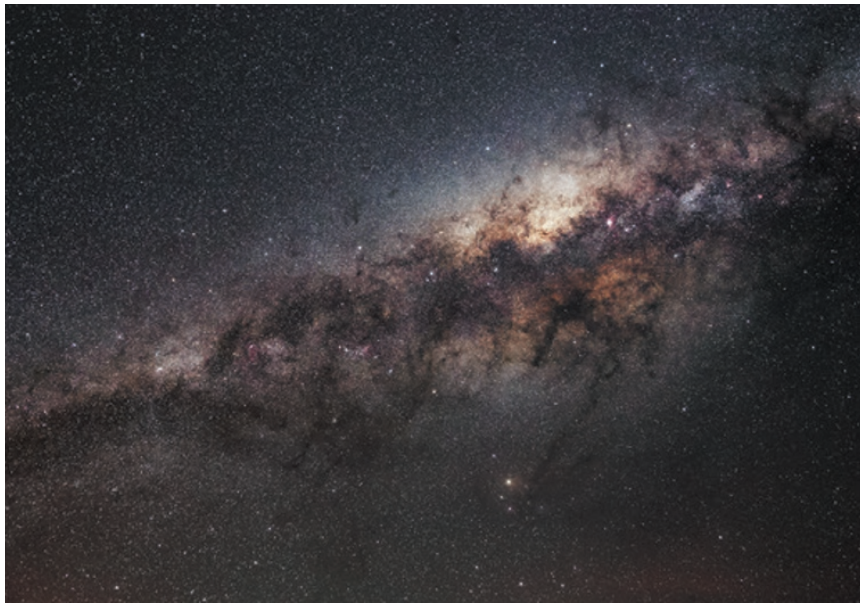

flight, anchoring the insects to the water.

Keeping four of their six legs on the water, the insects use the fluid's surface tension to support their body weight and move by flapping their hind wings, cruising along the water's surface at speeds of up to half a metre per second. Moving any faster would render them airborne, because the ripples they produced on the water would release their anchors.

J. Exp. Biol. 219, 752-766 (2016)

\section{ASTRONOMY}

\section{Milky Way's bulging waistline}

The mass of stars in the Milky Way's central bulge (pictured) is about 20 billion times the mass of the Sun - a much higher estimate than in most previous studies.

The central bulge protrudes from the Galaxy's main disk like the yolk of a fried egg and hosts a large density of stars. To estimate the mass of those stars, Elena Valenti at the European Southern Observatory (ESO) in Garching, Germany, and her team used a catalogue of stars of a particular type. The data are from ESO's Visible and Infrared Survey Telescope for Astronomy (VISTA) at the Paranal Observatory in Chile. The team also did a deeper study of all the stars in a small region of the bulge, in part using the Hubble Space Telescope.
Valenti says that this is the first study of the bulge based entirely on observation, without the help of theoretical models. Astron. Astrophys. 587, L6 (2016)

\section{LONGEVITY}

\section{Genetic switches for long life}

Researchers have homed in on the genetic control points that allow nematodes to live longer when they are on a low-calorie diet.

A team led by Jing-Dong Han of the Chinese Academy of Sciences in Shanghai analysed gene-expression changes over time in the nematode Caenorhabditis elegans. The worms were subjected either to caloric restriction or to intermittent fasting, both of which extend worm lifespan.

The team identified changes in the expression of various genes at different times, with metabolism genes responding early during the diet, and those linked to cell division and DNA repair changing later on. The researchers then used an algorithm to identify three sets of genes that regulate this altered expression. Changing the activity of the pathways controlled by these genes extended the lifespan of the worms, mimicking the effect of dietary restriction.

Cell Metab. 23, 529-540 (2016)

\section{DNATURE.COM}

For the latest research published by Naturevisit:

www.nature.com/latestresearch 